\title{
Long-term analysis of GOME in-flight calibration parameters and instrument degradation
}

\author{
Melanie Coldewey-Egbers, ${ }^{*}$ Sander Slijkhuis, Bernd Aberle, and Diego Loyola \\ German Aerospace Center (DLR), Remote Sensing Technology Institute (IMF), \\ Münchner Strasse 20, D-82234 Wessling, Germany \\ ${ }^{*}$ Corresponding author: Melanie.Coldewey-Egbers@dlr.de
}

Received 27 March 2008; revised 22 July 2008; accepted 24 July 2008;

posted 7 August 2008 (Doc. ID 92891); published 9 September 2008

\begin{abstract}
Since 1995, the Global Ozone Monitoring Experiment (GOME) has measured solar and backscattered spectra in the ultraviolet and visible wavelength range. Now, the extensive data set of the most important calibration parameters has been investigated thoroughly in order to analyze the long-term stability and performance of the instrument. This study focuses on GOME in-flight calibration and degradation for the solar path. Monitoring the sensor degradation yields an intensity decrease of $70 \%$ to $90 \%$ in $240-316 \mathrm{~nm}$ and $35 \%$ to $65 \%$ in $311-415 \mathrm{~nm}$. The spectral calibration is very stable over the whole period, although a very complex interaction between predisperser temperature and wavelength was found. The leakage current and the pixel-to-pixel gain increased significantly during the mission, which requires an accurate correction of the measured radiance and irradiance signals using proper calibration parameters. Finally, several outliers in the data sets can be directly assigned to instrument and satellite anomalies. (C) 2008 Optical Society of America
\end{abstract}

OCIS codes: $\quad 010.1290,120.6200,280.4991,300.6190$.

\section{Introduction}

The Global Ozone Monitoring Experiment (GOME) was launched on 21 April 1995 onboard the second European Remote Sensing Satellite (ERS-2). GOME is a nadir-viewing spectrometer covering the ultraviolet (UV) and visible wavelength range from 240 to $790 \mathrm{~nm}$ with moderate spectral resolution of 0.2 to $0.4 \mathrm{~nm}$ [1]. Its primary objective is the determination of the amounts and distributions of atmospheric trace constituents, such as ozone, nitrogen dioxide, or bromine oxide. GOME is a double monochromator, which has as dispersing elements a predisperser prism combined with a holographic grating in each of the four optical channels. The earthshine radiance and solar irradiance spectra are recorded with four linear Si-diode arrays with 1024 spectral elements each, covering the wavelength regions of 240 to $316 \mathrm{~nm}$ (channel 1), 311 to $405 \mathrm{~nm}$ (channel 2), 405 to $611 \mathrm{~nm}$ (channel 3), and 595 to $793 \mathrm{~nm}$ (channel

0003-6935/08/264749-13\$15.00/0

(C) 2008 Optical Society of America
4). Part of the light is branched out and recorded with three fast broadband silicon diodes, the polarization measurement devices (PMDs). These approximately cover the spectral ranges of channels 2,3 , and 4 , respectively. They measure the amount of light polarized parallel to the instrument slit, which is perpendicular to the plane of incidence of the scan mirror. GOME orbits the Earth at an altitude of about $790 \mathrm{~km}$ in a Sun-synchronous polar orbit; the local equator crossing time is about 10:30 UTC. Each orbit takes $\sim 100 \mathrm{~min}$ and ERS-2 completes $\sim 14$ orbits per day.

The GOME Data Processor (GDP) is the ground segment for the GOME instrument [2], incorporating, among other things, a level 0-to-1 processing chain and the complete GOME data archive. During the level 0-to-1 processing, GOME data is converted into calibrated radiances by applying calibration algorithms and calibration parameters [3]. Many calibration parameters are established on a regular basis from in-flight observations of the calibration lamp, of the internal light emitting diodes (LEDs), of the Sun, and from measurements under dark 
conditions. In addition, data from preflight instrument calibration and characterization (e.g., the polarization characteristics of the optical chain) is required. At the end of 2006, an update of the GDP level 0-to-1 processor has been developed in order to reprocess the entire GOME data set and to provide an homogeneous data record. The main driver for this updated version was the new Sun mean reference spectrum intensity check, and the associated closing of the time gaps between Sun mean reference spectrum updates on the level $1 \mathrm{~b}$ product (the check was meant to discard vignetted reference spectra, but it discarded good spectra, too, after degradation became too large). This opportunity has been used to include other algorithm developments as well, such as an extension of the GOME in-flight calibration parameter database and a modified spectral calibration, which resulted in a significantly improved wavelength stability over the entire time period, especially in channels 3 and 4 [4].

After almost 12 years of successful operation and data processing, it is now of great interest to analyze the in-flight calibration results and parameters to obtain a first long-term monitoring of their behavior. The calibration parameters that are calculated during the level 0 -to- 1 processing are a good means to monitor the stability of the instrument and its measurements. Stable long-term satellite data are needed as the requirements for more accurate satellite information products are steadily increasing, e.g., global climate monitoring requires temperature changes of a few tenths of a degree Celsius, or ozone trends with a precision of $1 \%$ per decade.

The outline of the paper is as follows. Section 2 contains an analysis of the time series of the Sun mean reference spectra and the PMD characteristics over the entire GOME period. The stability of the wavelength calibration is investigated in Section 3 , where a correlation analysis between the temperature and the wavelengths also has been performed. Section 4 is a summary of all dark signal trends, such as the leakage current and its noise for different integration time patterns, and the PMD offset. Furthermore, the influence of the South Atlantic Anomaly on those measurements has been estimated. Finally, the temporal development of the pixel-to-pixel gain correction is presented in Section 5.

\section{Sun Mean Reference Spectra and PMD Signals}

Once per day, GOME measures a short series of solar irradiance spectra with the sunlight reaching the scan mirror via the diffuser. During the measurements, the incidence angle on the diffuser is constant in azimuth (varies only with time of the year) and varies in elevation as the Sun moves through the field of view of the solar port. The incidence angle on the scan mirror is $41^{\circ}$, which is more or less representative for the nadir measurements that cover $49^{\circ} \pm 15^{\circ}$. All measurements within an elevation angle of $\pm 1.5^{\circ}$ with respect to the center are averaged and corrected for the azimuth dependence of the dif- fuser bidirectional scattering distribution function (BSDF) as calibrated on-ground. This yields the daily Sun mean reference spectrum, which is stored in the calibration database.

It is well known that optical components subjected to prolonged exposure to the space environment exhibit degradation of the optical properties. The GOME sensor shows degradation in all wavelength regions due to damages in its optical path. Figure 1 shows the ratios of the Sun mean reference spectra from 9 January 1997 to 2006 to the corresponding spectrum from 1996 . In channel 1 , the intensity decreased by $70 \%$ to $90 \%$. The decrease in channel 2 is still $35 \%$ to $65 \%$, whereas the degradation in channels 3 and 4 is lower. In channel 3 , the decrease started in 2001, when the GOME Sun measurements were additionally affected by an ERS-2 pointing problem (vignetting of the solar beam may occur when the Sun is near its extreme azimuth angles and mispointing directs it to the edge of the irradiance port's field of view). The gray shaded areas in Fig. 1 mark features caused by the dichroic filter that separates GOME channels 3 and 4 . The large amplitude in the channel 3-4 boundary is caused by a shift in the channel-separation wavelength. The transmission increase in channels 3 and 4 in the early part of the mission is probably also related to changes in the multilayer coating of this filter (outgassing). The oscillating behavior seen in all channels is the result of the etalon effect, which is caused by the gradual buildup of an ice layer on the GOME detectors and which leads to a spectral interference pattern $[\underline{5}, \underline{6}]$.

Figure 2 shows all Sun mean reference spectra of GOME from July 1995 to June 2006 for four single wavelengths $(290 \mathrm{~nm}$-channel $1,330 \mathrm{~nm}$-channel $2,430 \mathrm{~nm}$-channel 3 , and $760 \mathrm{~nm}$-channel 4) used in the retrieval of ozone, $\mathrm{NO}_{2}$, and cloud information [7]. Thin curves denote uncorrected data. The low periodic variation is due to the seasonality of the Sun-Earth distance, which is maximum in July and minimum in January. Large peaks in the time series for all wavelengths at the beginning of 2001 are due to severe problems with the ERS-2 spacecraft. They can be directly assigned to data gaps and GOME anomalies, such as instrument

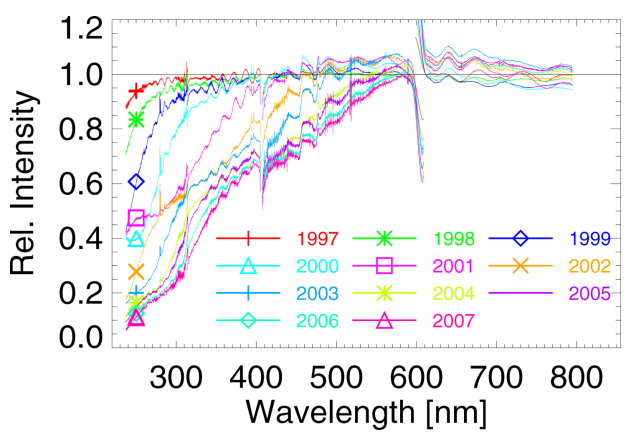

Fig. 1. (Color online) Ratios of the Sun mean reference spectra of GOME from 9 January 1997 to 2007 to the corresponding reference spectrum from 1996. 


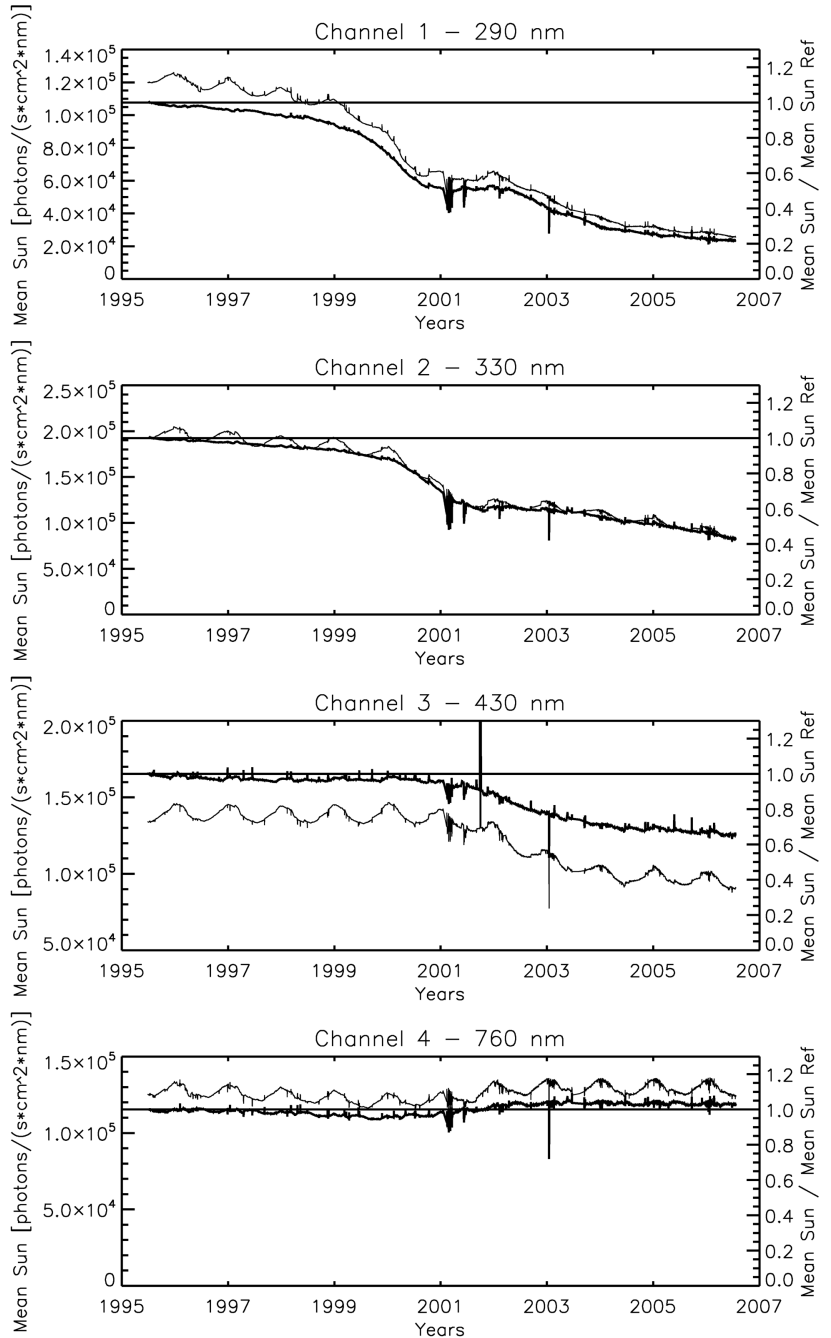

Fig. 2. Sun mean reference intensities of GOME from July 1995 to June 2006 for four single wavelengths (from top to bottom: $290 \mathrm{~nm}$-channel $1,330 \mathrm{~nm}$-channel $2,430 \mathrm{~nm}$-channel 3 , and $760 \mathrm{~nm}$ - channel 4). Thin curves denote uncorrected data. The low periodic variation is due to the seasonality of the Sun-Earth distance, which is maximum in July and minimum in January. Peaks can be assigned to GOME anomalies and to the etalon effect. Thick curves (right $y$ axis) denote normalized Sun mean reference data, which are corrected for etalon and Sun-Earth distance.

switchoffs, as regularly documented in the GOME yearly anomaly reports [8]. Besides the large peaks, several small peaks can be identified in the curves, which occur for different wavelengths at different dates. Therefore, they cannot be explained with instrument anomalies, which would affect all channels simultaneously, but with changes in etalon structure.

The thick curves in Fig. 2 denote the Sun mean reference data, which are first corrected for the etalon effect according to

$$
\text { etalon }(t, \lambda)=\frac{\operatorname{Sun}(t, \lambda) / \operatorname{Sun}\left(t=t_{\mathrm{ref}}, \lambda\right)}{\operatorname{poly}_{\lambda}\left(\operatorname{Sun}(t, \lambda) / \operatorname{Sun}\left(t=t_{\mathrm{ref}}, \lambda\right)\right)},
$$

where $\operatorname{poly}_{\lambda}$ is a third-order polynomial in wavelength, $\operatorname{Sun}\left(t=t_{\mathrm{ref}}, \lambda\right)$ is the selected reference spec- trum from 3 July 1995, and $\operatorname{Sun}(t, \lambda)$ are all available Sun mean reference spectra until June 2006. The corrected Sun spectrum is then given by

$$
\operatorname{Sun} \_ \text {new }(t, \lambda)=\operatorname{Sun}(t, \lambda) / \text { etalon }(t, \lambda) \text {. }
$$

Second, all spectra are normalized to 1 astronomical unit (AU) in order to remove the seasonal dependence. Finally, they are normalized to the intensity of the reference spectrum from 3 July 1995 to calculate the percentage decrease. The intensity decreased by $80 \%$ at $290 \mathrm{~nm}$ and by $60 \%$ at $330 \mathrm{~nm}$ until June 2006. The decrease in channel 3 $(430 \mathrm{~nm})$ started in 2001 and has now reached almost $40 \%$. In channel 4 , at $760 \mathrm{~nm}$, only minor changes are observed: a slight decrease of $10 \%$ from 1995 to 2001 and then a short increase of 5\% until 2006.

The corresponding time series for the three PMD signals are depicted in Fig. 3. Again, thick curves denote the signals normalized to $1 \mathrm{AU}$ in order to eliminate seasonality and to the reference measurements from 3 July 1995. The degradation of the PMD signals show almost the same behavior as for the corresponding channel wavelengths. As for the Sun mean reference spectra, the large outliers can be assigned to instrument anomalies [8].
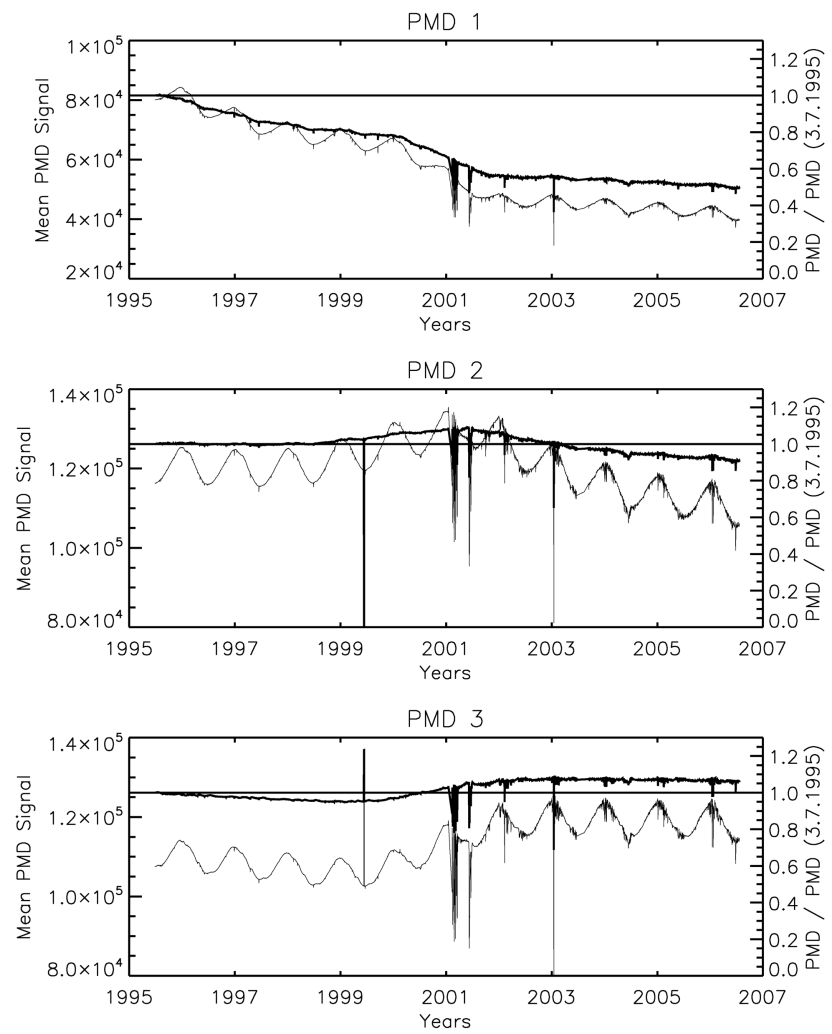

Fig. 3. PMD signals measured by GOME between July 1995 and June 2006. From top to bottom: PMD 1, PMD 2, and PMD 3. Thin curves denote uncorrected data, thick curves (right $y$ axis) denote data normalized to $1 \mathrm{AU}$ and to the reference signal from 3 July 1995. 


\section{A. PMD $Q$-Factors}

The polarization state of the incoming light is measured using three fast broadband PMDs with bandwidths approximating the spectral range of channels 2,3 , and 4 . The integration time of the PMD measurements is appointed to $93.75 \mathrm{~ms}$, i.e., $16 \mathrm{PMD}$ measurements are available for one detector channel measurement at the default $1.5 \mathrm{~s}$ integration time. One fractional polarization value $p$ is evaluated for each PMD, using the ratio of the PMD signal to channel signals from the same wavelength region, while assuming that the fraction of intensity polarized parallel to the instrument slit, $p$, is constant for that wavelength range [3]. Assuming that the Sun should yield an unpolarized signal with $p=0.5$, a calibration constant called the " $Q$-factor" may be calculated, which transforms a measured solar signal with fractional polarization $p$ to an unpolarized signal with $p=0.5$.

The $Q$-factors are defined as the relative difference between the measured solar signal of PMD $i$, with $i=1,2,3$, and the expected PMD signal calculated from the corresponding channel signals when unpolarized input is assumed:

$$
Q \text {-factor } i=\left(\mathrm{PMD}_{i}-\sum_{j} X_{j} \times \text { channel }_{j}\right) / \mathrm{PMD}_{i},
$$

where channel ${ }_{j}$ is the channel signal of pixel $j$ and $X_{j}$ is the ratio of the PMD signal to the channel signal for a monochromatic input signal, as obtained from on-ground calibration measurements. The $Q$-factors thus incorporate the differential degradation between PMD signals and corresponding channel signals, since the time of on-ground calibration. They also incorporate any calibration errors (including straylight) in the on-ground calibration. Including the $Q$-factors in operational processing provides a self-calibration without the need of explicitly knowing errors or changes in key data.

The time series of the $Q$-factors for each PMD are shown in Fig. 4, from July 1995 to June 2006. The strong decrease of $Q$-factor 1 is connected to the different degradation of the PMD 1 signal and the measured signal in channel 2. The PMD decreases faster compared to the channel up to the year 1999 and then from 2001 to 2006 the channel signal decreases faster. $Q$-factor 2 increases slowly from 1995 to 2006; that means the PMD 2 signal degrades less than the average signal in channel 3 (see also Figs. $\underline{2}$ and $\underline{3}$ ). $Q$-factor 3 is more or less stable ( $\sim 0.15$ to 0.2$)$ over the entire period. Measurements carried out during the calibration of the GOME Flight Model have shown that all three PMDs are sensitive to light above $790 \mathrm{~nm}$. Early in-flight solar data showed that straylight appears to be worst in PMD $3(\sim 13 \%)$. This explains why $Q$-factor 3 is nonzero already, before the onset of degradation. As for the Sun mean reference data and the PMD signals, the irregular large peaks in all three curves are due to GOME anomalies, such
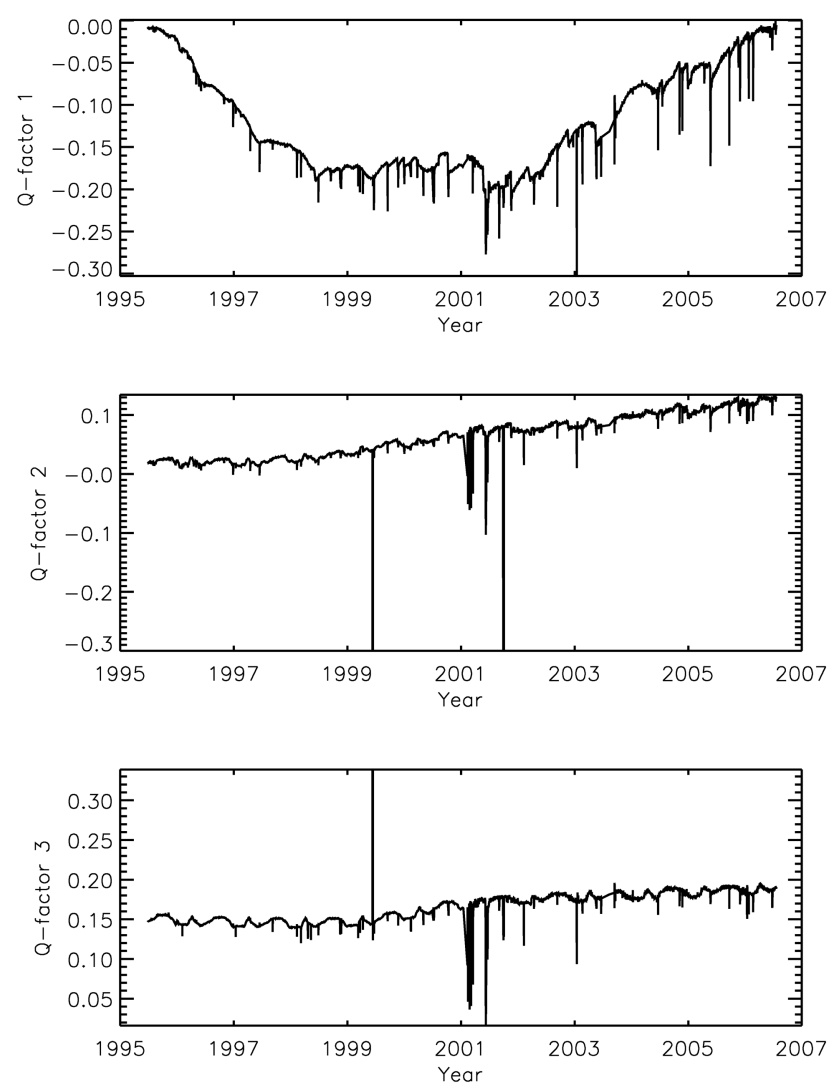

Fig. 4. GOME PMD $Q$-factors from July 1995 to June 2006. From top to bottom: $Q$-factor 1, $Q$-factor 2, and $Q$-factor 3 .

as cooler switchoffs, instrument or satellite switchoffs, onboard anomalies, or special operations []].

\section{B. Degradation Modeling}

A detailed modeling of the observations is out of scope of this paper. However, it may be of interest to place the observations in the perspective of previously published work.

There are several reasons to believe that the scan mirror is the main degrading optical element. The similarity of PMD degradation to main-channel degradation suggests a cause early in the optical path. Degradation of the Sun diffuser, which is the main degrading element in, e.g., the Nimbus-7 SBUV instrument [9], is not observed [10], at least not until July 2003, when the quality of diffuser-monitoring measurements (based on measurements of the spectral calibration lamp over the diffuser) suffered after failure of the onboard tape recorder. The stability of the diffuser came as a surprise to many, but note that, in GOME, the Sun diffuser is protected by a shutter and solar intensity is further reduced by a mesh in front of the diffuser; furthermore, the exposure to solar observations is limited to a few minutes per day. The third reason is an observed scan-angle dependence of the degradation. This is seen as a somewhat different degradation of lunar measurements with respect to solar measurements-the difference in optical path is only in the Sun diffuser (which is found to be stable) and a different incidence 
angle on the scan mirror. Furthermore, in the earthshine spectra, an east-west difference that increases with time can be observed.

The degradation in channels 1 and 2 has been explained in terms of deposits on the scan mirror [11]. The aluminum surface of the scan mirror is protected with a layer of $\mathrm{MgF}_{2}$. This layer also gives rise intentionally to an interference effect, which increases the UV reflectivity of the mirror. Deposits in or on this layer not only cause a smooth degradation of reflectance that increases toward shorter wavelengths, but also change the interference pattern. This can explain features in Fig. 1, such as the upturn in reflectivity around $250 \mathrm{~nm}$ in 2001, which, in later years, progresses to longer wavelengths.

Deposits on the scan mirror will not only change its reflectivity, but also its polarization properties. The influence on the polarization correction of the earthshine radiance has not been modeled. However, the influence on retrieved polarization values from the PMDs is kept to a minimum by the self-calibration procedure using the the PMD $Q$-factors derived from solar measurements. The validity of the approach has been discussed in [12]. They found a scan-angle dependence, with the polarization values remaining stable for the west pixels in the scan, but degrading for the east pixels.

Mirror reflectivity for perpendicular polarization, which is the polarization component measured by the PMDs, is expected to degrade less than the reflectivity for unpolarized light. This may explain why the PMD signal degrades somewhat less than the channel signal (at least after 2001).

Although the model of [11] gives a qualitative explanation of the degradation, it does not model the observed degradation in every detail. It is, therefore, not (yet) up to the standard that it may be used for the degradation correction of the measurements.

A degradation correction algorithm based on the measured solar spectra has been developed in the framework of the GOME Data Quality Improvement (GDAQI) project [13]. Since, for the atmospheric trace gas retrieval, the ratio of earthshine to solar spectra is used, a scan-angle dependent degradation may result in nonnegligible errors. This is especially true for retrieval that uses absolute radiance, such as ozone profile retrieval.

To improve the situation for the latter, a study has been performed at the Netherlands Institute for Space Research (SRON) [14] to correct for the degradation in reflection. In the GDP level 0-to-1 software, starting at version 3.0, options exist to correct both the solar degradation and the reflection degradation. The latter is currently available only for measurements before 2003, is spectrally resolved up to $350 \mathrm{~nm}$, and represents an average value for the complete swath width, as covered by channel 1 measurements. The reflection correction relies on a radiative transfer model in combination with ozone sonde data.
The operation level 2 retrieval algorithm for total column amounts of trace gases does not use absolute radiometric calibration and is, therefore, insensitive to this kind of degradation. Ground-based validation has confirmed the over 10 year stability for ozone, nitrogen dioxide, and bromine oxide $[15,16]$.

\section{Spectral Calibration}

To assign a certain wavelength to each individual GOME detector pixel, the instrument houses a platinum-chromium-neon hollow cathode emission lamp, which provides a sufficient number of atomic emission lines of the three elements [17]. Because of well-known spectral positions of these lines, the spectrometer can be calibrated during flight. Several lamp spectra are measured over the orbit approximately once per month, when the calibration timeline is run for five orbits. In addition, a spectral calibration is performed every day just before and after the Sun calibration. Those measurements are available until April 1998, when the daily calibration was reduced to only one measurement sequence per day. Since September 2001 the calibration lamp has been used only during the five orbits of the monthly calibration, which is the result of numerous lamp failures, when the voltage has not reached its nominal value. The corresponding wavelength calibration parameters are stored in the calibration database as a function of temperature measured at the predisperser prism. In operational processing calibration parameters are then selected for each measurement from the database according to the predisperser temperature. In terms of spectral shift, this prism is the most temperature-sensitive element of the optical system [18]. Each individual GOME spectrum is, thus, implicitly corrected for temperature variations that are caused by seasonal variations, the position in orbit, and by the rate of degradation of thermally sensitive optical elements [see also Fig. 7(a)].

The calibration parameters are obtained by fitting a polynomial through the pixel-wavelength pairs from the lamp measurements [3]. Sixty-seven emission lines have been selected within the spectral range from 240 to $790 \mathrm{~nm}$ that is covered by GOME. For each individual line, the pixel-number centers of the maximum intensity, the statistical moments, and the full width at half-maximum (FWHM) are computed. Those values have to fulfill certain statistical criteria for a line to be accepted: the signal intensity must be larger than a given minimum, the FWHM must be larger than a certain value, and the line must be roughly symmetric. The thresholds for these criteria have been determined during the preflight measurements and the commissioning phase of GOME.

At least seven spectral lines in each GOME channel are required for the polynomial fitting. Thirdorder polynomials for channels 1 and 2, and fourth-order polynomials for channels 3 and 4, respectively, are fitted through the pixel-wavelength 
pairs using the singular value decomposition algorithm [19].

\section{A. Stability}

In the framework of the ESA project "Long-Term Monitoring of GOME Calibration Parameters" [20], nine of those 67 spectral emission lines were identified to be too unstable for an exact calibration analysis. The lines do not meet the well-defined statistical criteria for all available lamp measurements. As a consequence, the polynomial parameters and the wavelengths themselves used to show jumps, depending on the number of spectral lines per channel that were used for the analysis. Finally it was recommended to exclude those emission lines in order to stabilize the whole calibration process.

Wavelength calibration accuracy may be inferred from the differences between the exact spectral position of the emission lines and the wavelengths described by the polynomial fit. Figure 5 shows the standard deviation of the wavelengths of all emission lines over the entire period (all available calibration orbits) between June 1995 and May 2003 for the old and for the new calibrations. Furthermore, those nine unstable spectral lines that were removed from the analysis are marked. The largest changes can be found at the beginning of channel 3 , where three lines were excluded, and at the end of channel 4 around $760 \mathrm{~nm}$, where the very unstable last line has been removed. The latter region is important for cloud detection [21], which may, therefore, be expected to be improved in the new GDP products. In general, the wavelength calibration is much more stable now using the new line selection.

Another way to look at wavelength calibration stability is to examine the calibrated wavelength for the same predisperser temperature, measured at different times. The variability of all GOME wavelengths is presented in Fig. $\underline{6}$ for one fixed temperature of $281.9 \mathrm{~K}$, which is the medial value of all measurements. All wavelengths are very stable over the entire period. The variability is between 0.0015 and $0.004 \mathrm{~nm}$, except at the beginning of channel 3 and

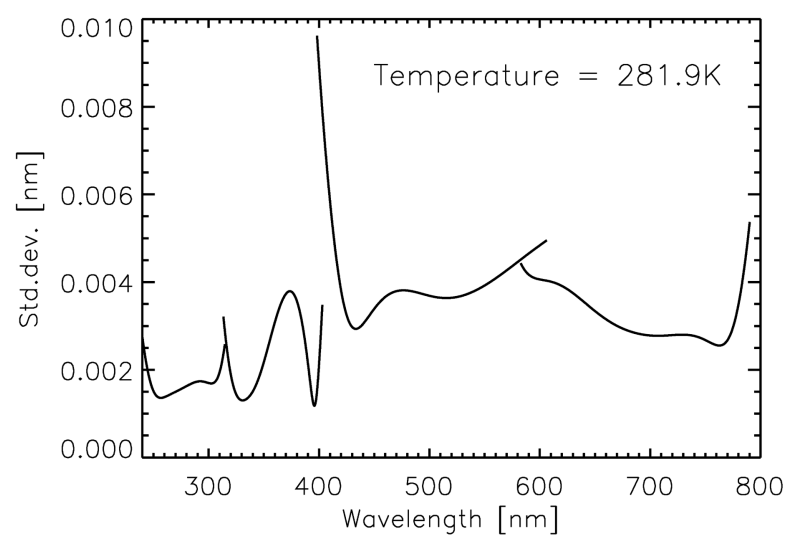

Fig. 6. Standard deviation of all GOME wavelengths for one fixed temperature of $281.9 \mathrm{~K}$, which is the medial value of all measurements.

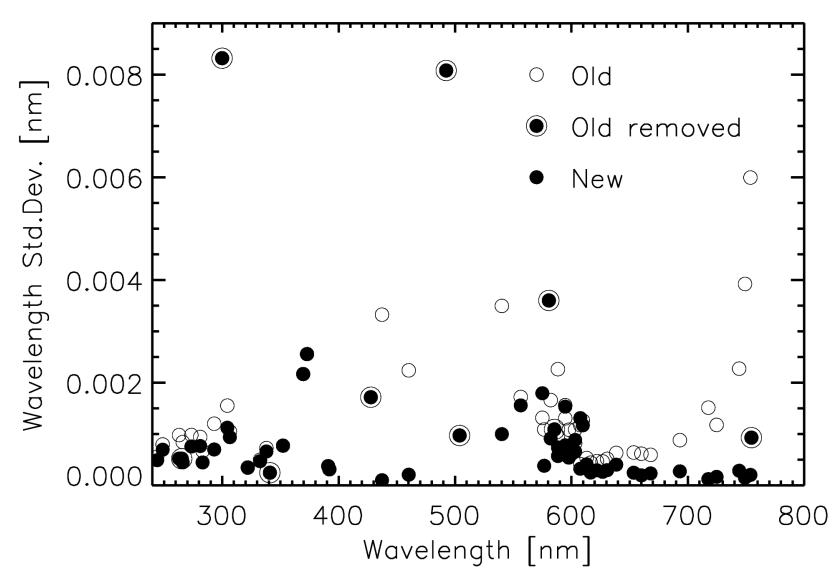

Fig. 5. Standard deviation of wavelengths for all calibration orbits between June 1995 and May 2003. Open circles: old wavelength calibration with 67 spectral lines; solid dots surrounded by circles: spectral lines, which were removed from the calibration analysis; and solid dots: new wavelength calibration.

at the very end of channel 4 , where it is larger due to the lack of enough emission lines in these spectral regions. These results are comparable to the investigations by [22], who found values for the variations in time of the wavelength calibration from 0.0015 to $0.0034 \mathrm{~nm}$ for nine narrow spectral bands.

This achieved accuracy approaches the required accuracy for differential optical absorption spectroscopy spectral fitting of trace gases, which in the literature is cited as $1 / 50$ to $1 / 100$ of a pixel [23]; the dispersion of GOME is around $0.1 \mathrm{~nm}$ per pixel in channels 1 and 2 and around $0.2 \mathrm{~nm}$ per pixel in channels 3 and 4 . Note that, during the operational calibration process, the accuracy of the wavelengths is further improved by utilizing the cross-correlation algorithm [24]. Here, a representative subset of GOME solar measurements from the whole lifetime is taken, to which the cross correlation is applied. The retrieved polynomial coefficients are then added to the polynomial coefficients of the wavelength calibration from the spectral lamp measurements.

\section{B. Temperature Dependence}

One of the key elements in the optical system of GOME is a quartz predisperser prism. The refractive index of quartz depends not only on the wavelength of the light passing through it, but also on the temperature of the prism, which is, in fact, the most temperature-sensitive element of the whole optical system [18]. It is expected, that the temperature increases along an orbit, partly due to warming of the satellite by the Sun and partly because light passes through the instrument [22]. Those temperature changes may, therefore, affect the wavelength calibration from the lamp measurements. Figure 7(a) shows the time series of the temperature measured at the predisperser prism. From 1995 to 2006, a small increase of $\sim 2.5 \mathrm{~K}$ can be observed, which is due to the degradation of the thermal system. The regular peaks on the series denote the temperature changes 


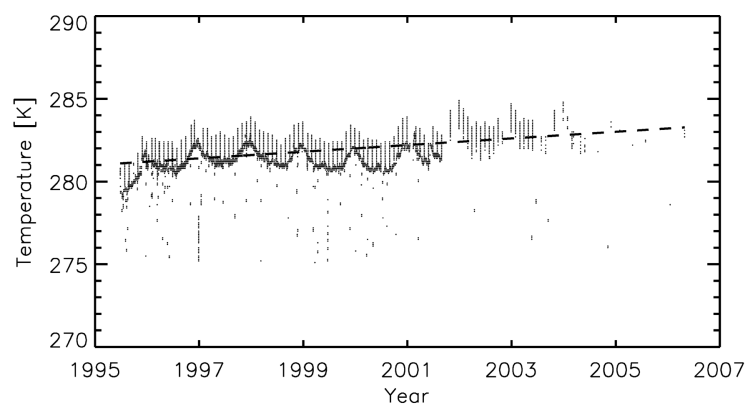

(a)

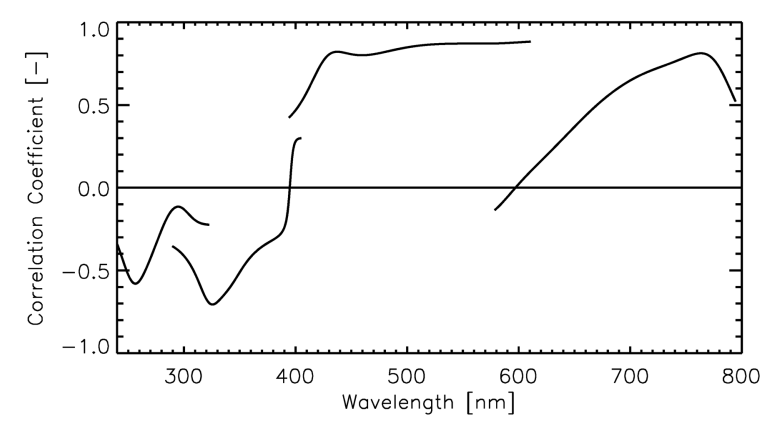

(b)

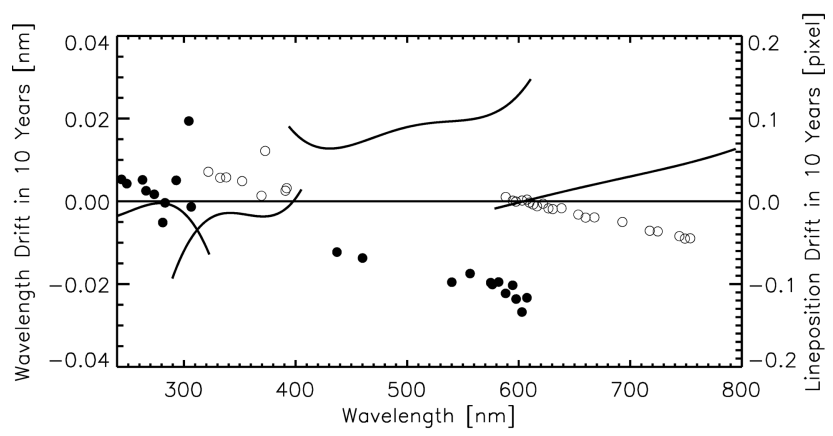

(c)

Fig. 7. (a) Temperature at predisperser prism as a function of time, dashed line: linear fit. (b) Correlation coefficient between temperature (with $T>279 \mathrm{~K}$ ) and wavelength for all GOME calibration orbits until June 2001. (c) Wavelength drift (solid curves, left $y$ axis) and line position drift (solid dots and open circles, right $y$ axis) after $\sim 10$ years (55.000 orbits). Solid dots correspond to channels 1 and 3, open circles correspond to channels 2 and 4, respectively.

that occur during the monthly calibration sequence. They can reach up to $1.5 \mathrm{~K}$ within five or six consecutive orbits. Furthermore, a seasonal cycle exists, with maximum values in December/January when the Sun-Earth distance is at a minimum. Outliers are connected to instrument and cooler switchoffs.

The following discussion focuses on the correlation coefficient between temperature and the wavelength as determined from the polynomial fitting. It is depicted as a function of wavelength for all four channels in Fig. 7(b). In channel 3 and at the end of channel 4 a significant positive correlation between both parameters is found, whereas, in the other spectral regions, it is negligible ( $\rho< \pm 0.5)$, but mainly negative. Parts of these results were also found in a study by [22]. He investigated the correlation between the predisperser prism's temperature and the wavelength change along orbits for nine small spectral windows of 1 to $20 \mathrm{~nm}$ width, using a different wavelength calibration method, as described in [25]. The windows cover the end of channel 1, channel 2 , and the end of channel 4. Although this study was limited to three days (43 orbits) in March 2000, the insignificant, but predominatly negative, correlation in channels 1 and 2 and the positive correlation at the end of channel 4 were also found. Channel 3, where we find the largest correlation, was unfortunately not part of his investigation.

Additionally, wavelength trends with time have been calculated for each GOME wavelength. The analysis is based on the data from the 6 year period between June 1995 and June 2001. The estimated drift has then been extrapolated to values expected for a 10 year period of about 55,000 orbits, which is presented in Fig. 7(c). The drift depends strongly on the position on the detectors. It is below $0.01 \mathrm{~nm}$ in channels 1,2 , and 4, except for the overlap region of channels 1 and 2 . The wavelength change in channel 3 is $\sim 0.02 \mathrm{~nm}$ in 10 years. Additionally, the corresponding trend of the line positions of the 51 spectral lamp lines is shown. Their drift is anticorrelated to the wavelength drift, as expected.

These wavelength changes may be related to changes of the thermal environment during the lifetime of GOME. In the case of a significant correlation between wavelength and temperature (e.g., in channel 3), a drift in the wavelengths is detected, as well as an annual cycle (without figure), which corresponds to the temperature time series. Thermally induced wavelength changes were also found for other backscatter UV instruments, such as the Total Ozone Mapping Spectrometer (TOMS) on Meteor 3 [26] and the Shuttle Solar Backscatter Ultraviolet (SSBUV) experiment $[27,28]$.

As the wavelength stability for a given temperature is very high (see Fig. 6), this implies that there is a very complex interaction between temperature and spectral position on the detector.

\section{Leakage Current and PMD Offset}

The detectors used for the four channels of the GOME sensors are random-access linear photodiode arrays. One characteristic of these devices is a certain amount of leakage current produced by thermal leakage. To keep this current as low as possible, Peltier elements attached to the diode arrays and connected to passive deep space radiators actively cool the detectors thermoelectrically to $\sim 235 \mathrm{~K}$. It was expected that the leakage current might depend on the orbital position of the satellite and the lifetime of the instrument (sensor degradation). Also, it is well known that radiation damage caused by hits of highenergetic protons may result in permanent damage in the form of a significant increase in leakage current or random telegraph signals (RTS), where the pixel signal jumps between multiple energy levels. 
Additionally, nonpermanent damage may be caused by spikes as observed in the South Atlantic Anomaly region (see Section 4.A). Therefore, it is necessary to continuously monitor the leakage current spectra and associated noise for each channel. This is done by dark measurements taken every orbit on the eclipse side. From these measurements the charge readouts of all detector pixels can be corrected for any leakage contribution. As the PMD detectors are nonintegrating devices, they do not have a leakage current. Nevertheless, those detectors must be corrected for their zero offsets and also their noise level must be properly monitored.

During the commissioning phase, it was found that a certain amount of cross talk is present, depending on the integration time pattern of the four detectors. Therefore, the dark-side measurements have to be taken with the same integration time patterns as those employed for scanning and other calibration measurements. This requires at least 10 consecutive dark measurements $S_{i}^{\text {dark }_{j}, k}$ with $j=1, \ldots, 10$ measurements and $i=1, \ldots, 1024$ detector pixels for each time pattern $k$ from which a mean spectrum of the leakage charge signal is calculated $\overline{S_{i}^{\text {dark, }, k}}$. The leakage current correction is then simply the subtraction of this spectrum from the measured signal $S_{i}^{\text {meas }, k}$ with the same integration time pattern $k$ :

$$
S_{i}=S_{i}^{\text {meas }, k}-\overline{S_{i}^{\text {dark }, k}}
$$

with

$$
\overline{S_{i}^{\text {dark }, k}}=\frac{1}{n} \sum_{j=1}^{n}\left(S_{i}^{\text {dark }, k}\right)_{j}
$$

The time pattern $k$ describes the number of clock pulses, where one pulse takes $93.75 \mathrm{~ms}$; e.g., a time pattern of 640 is equivalent to $60 \mathrm{~s}$. Because of the low temperature $\left(-38^{\circ} \mathrm{C}\right)$ of the detector arrays, the time-dependent leakage current itself is very small $(\sim 1 \mathrm{BU} / \mathrm{s})$, but the complete dark signal is comprised of two parts, where the second part is a constant value of between 140 and $150 \mathrm{BU}$, which is called the fixed pattern readout noise. The noise on the signals of the detector pixel readouts is also expected to be very small and a constant over all detector pixels. All dark measurements (with the same integration time) from one orbit are used to calculate standard deviations for each detector pixel. The mean value of these standard deviations will yield the mean noise value for the detector pixel readouts.

For the following investigations, the three most representative patterns have been selected: time pattern $640(60 \mathrm{~s}$ for band $1 \mathrm{a}$, and $6 \mathrm{~s}$ for the other bands), which is used in the polar-view mode of GOME; time pattern 320 (30 s for all bands), which is used for the internal LED measurements; and time pattern 128 ( $12 \mathrm{~s}$ for band $1 \mathrm{a}$, and $1.5 \mathrm{~s}$ for the other bands with coadding included), which accounts for the normal scanning orbits. The database was analyzed for five years: 1996, 1997, 2000, 2003, and 2005.

The dark signal for integration time pattern 640 , its percentage increase as a function of time (1996 to 2005), as well as a simple linear-regression analysis, are presented in Fig. 8. A strong linear increase of the mean dark signal from 260 to $500 \mathrm{BU}(+80 \%)$ between 1996 and 2005 is found for channel 1 (band 1a), whereas the average increase in channels 2,3 , and 4 is around $15 \%$ to $20 \%$. The larger increase in channel 1 is due to the large integration time of $60 \mathrm{~s}$, compared to $6 \mathrm{~s}$ for the other channels. In channel 4, an abrupt decrease is detected for the second half of 2005 . The increase for pattern 320 , with a $30 \mathrm{~s}$ integration time, is around $60 \%$ for all channels. For time pattern 128 , an increase of $25 \%$ was found for band $1 \mathrm{a}$ with a $12 \mathrm{~s}$ integration time, whereas the increase is only $1 \%$ for the other bands with a short integration time of $1.5 \mathrm{~s}$.

As the increase of the dark signal (in BU) is proportional to the integration time, the increase between 1996 and 2005 was converted to BU/s. For all integration times similar values of 3.5 to $4.5 \mathrm{BU} / \mathrm{s}$ in 10 years were found which are maximum in channel 2. This is consistent with the analysis performed by
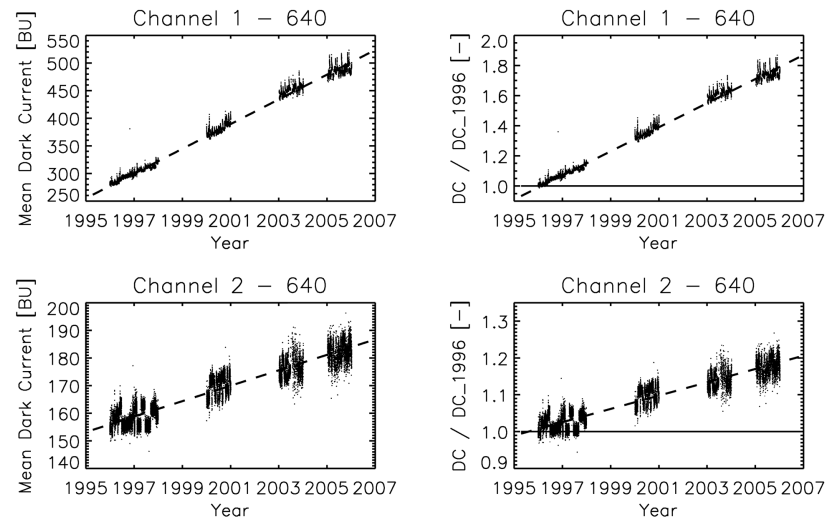

Year
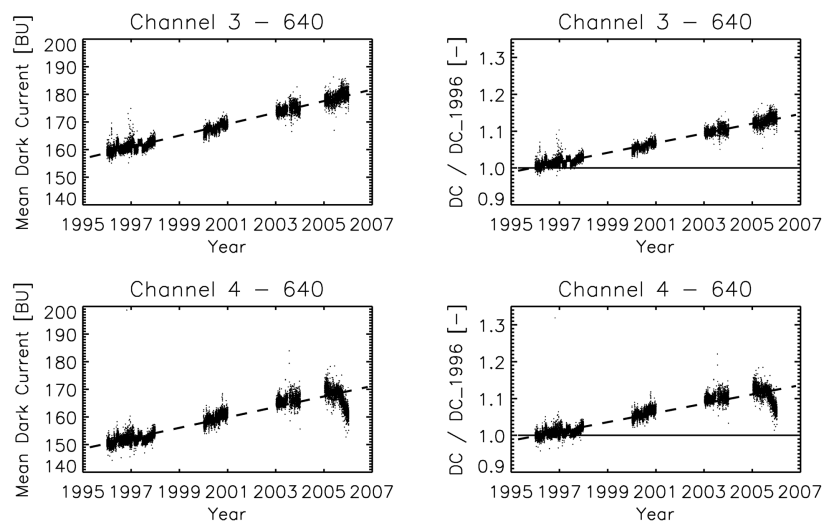

Fig. 8. Mean dark signal (left panels) and percentage increase of the leakage current since January 1996 (right panels) as a function of orbit number for integration time pattern 640 ( $60 \mathrm{~s}$ for band 1a and $6 \mathrm{~s}$ for the other bands). Data are plotted for five selected years: 1996, 1997, 2000, 2003, and 2005. From top to bottom: channel 1, channel 2, channel 3, and channel 4. Dashed lines denote a simple linear regression analysis. 
Dehn for the time period from June 1995 to June 2002 [29], which found values of $\sim 3 \mathrm{BU} / \mathrm{s}$ in 7 years.

To look for permanent damage in the form of RTS effects, the dark-current time series was also analyzed on an individual-pixel basis. The increase is more or less homogeneous over the channels, and no pixel signal jumps indicating RTS could be identified. However, for some spectral regions, changes in cross-talk effects were found for the longer integration time patterns.

The time series of the PMD offsets and the corresponding noise level are shown in Fig. 9. The offsets are around $1320 \mathrm{BU}$ for PMD 1 and around 510 BU for PMDs 2 and 3. All offsets increased slowly, by about $0.5 \%$ to $1 \%$, up to 2005 . Then, a small decrease

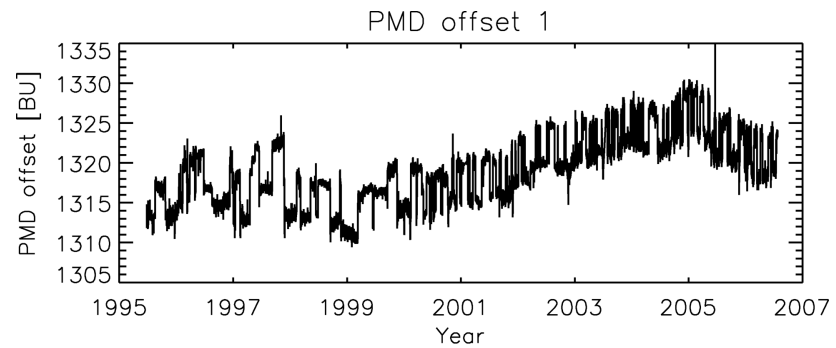

(a)

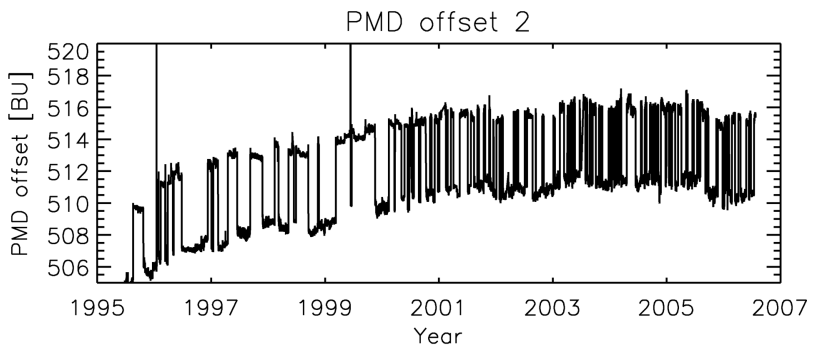

(b)

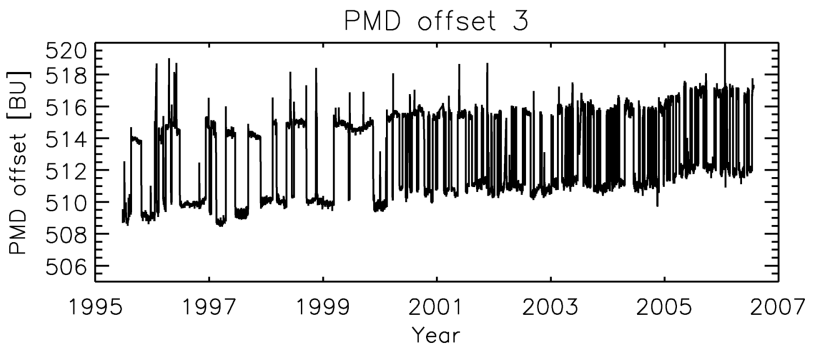

(c)

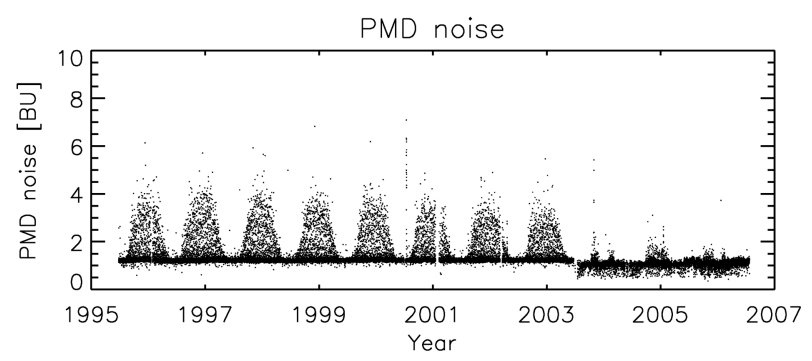

(d)

Fig. 9. PMD offsets and PMD noise level from July 1995 to June 2006. (a) PMD 1 offset, (b) PMD 2 offset, (c) PMD 3 offset, and (d) PMD noise level. can be detected. In addtion, periodical jumps of the order of 5 to $8 \mathrm{BU}$, which correspond to $0.5 \%$ for PMD 1 and $1.0 \%$ for PMDs 2 and 3, can be seen. They can be explained with GOME anomalies as instrument and cooler switchoffs, as well as onboard anomalies and special operations [8]. The PMD noise [Fig. 9(d)] is defined as the mean value of the standard deviations, which are calculated for each PMD over all 16 PMD measurements. It is around 1 and $5 \mathrm{BU}$ up to June 2003, and around $1 \mathrm{BU}$ afterward. This date marks the failure of the onboard tape recorder, after which no complete orbits could be transmitted to ground. In particular, data from inside the South Atlantic Anomaly region are no longer present. They seem to have a strong influence on the noise level, especially in the winter months when the EarthSun distance is at minimum.

\section{A. Influence of the South Atlantic Anomaly}

It has been known for more than 40 years that there is a weak geomagnetic field in the South Atlantic Ocean, known as the South Atlantic Anomaly (SAA). In this region, the Earth's inner van Allen radiation belt makes its closest approach to the surface, caused by the fact that the center of Earth's magnetic field is offset from its geographic center by a few hundred kilometers. This dip coincides with a region of intense radiation in space near the Earth, which may cause damage to spacecraft in low Earth orbit [30]. It is known that GOME on ERS-2 also shows some sensitivity to the space radiation environment. High-energy protons impact the detectors, leading to large data spikes, especially in band 1 , where the signal is low [31]. This may cause complications when those data are used during the calibration process. Once the spacecraft leaves the SAA, the increased signals disappear.

For the following study, the daily dark-side measurements of the leakage-current noise have been investigated. All GOME orbits crossing the SAA region-reaching approximately from the equator to $60^{\circ} \mathrm{S}$ and from $30^{\circ}$ to $60^{\circ} \mathrm{W}$-during night time were separated from those that do not cover this area. As the exact overpass geolocation data are not available for those dark-side calibration measurements, the following analysis must be considered simply as a qualitative estimation of the SAA influence. Because of the huge extent of the leakagecurrent database, only two years (1997 and 2000) and the three most representative integration time patterns $(640,320$, and 128) have been investigated. Figure 10 shows the leakage current as a function of pixel number in channel 4 for time pattern 320 for 10 consecutive orbits (9000 to 9009) in 1997. To highlight the differences, the consecutive measurements have been plotted with a constant offset of $-20 \mathrm{BU}$. The top curve belongs to orbit number 9000 and the bottom curve belongs to orbit number 9009 . Orbits 9002 and 9003 (third and fourth curves from top) pass over the SAA region. The measurements are much more noisy and they contain several large 


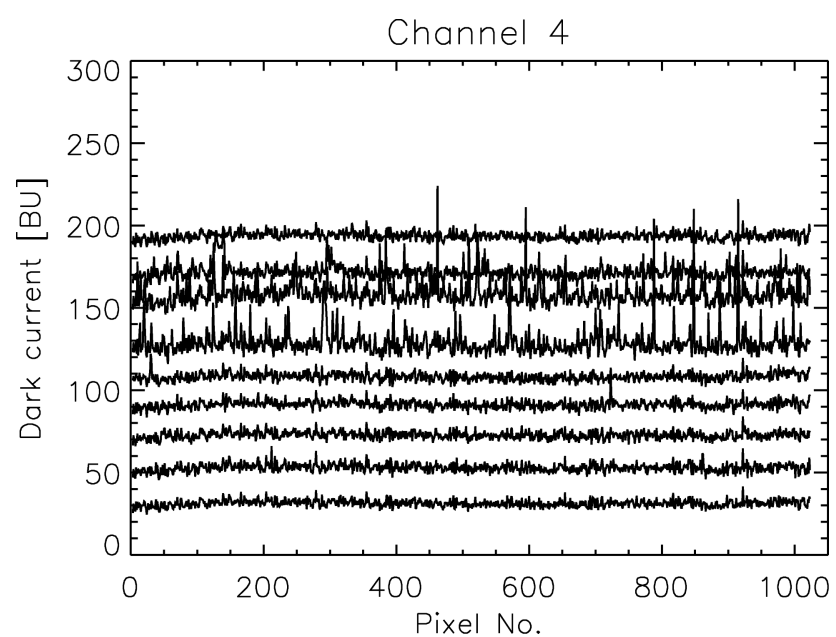

Fig. 10. Leakage current as a function of pixel number in channel 4 for time pattern 320 for 10 consecutive orbits (9000 to 9009) in the year 1997. To highlight the differences, the consecutive measurements have been plotted with a constant offset of $-20 \mathrm{BU}$. The top curve belongs to orbit number 9000 , and the bottom curve belongs to orbit number 9009 . Third and fourth curve from top (orbits 9002 and 9003) pass over the SAA region.

data peaks on individual pixels due to high-energy protons.

Figure 11(a) shows the noise of the dark-current measurements for time pattern 320 from 1995 to 2006. Again, as for the PMD noise, in June 2003 an abrupt change of the behavior is detected (average noise level decreases), when measurements from the southern hemisphere, especially from the SAA region, are no longer available. For Figs. 11(b) and 11(c), noise values from 1997 were separated for measurements outside and inside the SAA region and plotted as a function of month in 1997. The noise level inside the SAA region is enhanced and shows a distinct seasonal cycle, with maximum values in winter months.

The dark current itself is slightly larger inside the SAA than outside the SAA, which may be due to the expected spikes on individual detector pixels. Calculation of the dark signal using these measurements from inside the SAA may yield to an overestimation of the leakage and, therefore, to an underestimation of the real signal. The same analysis for the year 2000 and the other time patterns confirms these results (without figures). The influence of the SAA on the dark current and its noise level is largest for the long integration times (e.g., 30 and 60 s). It becomes smaller for the shorter ones of, e.g., $1.5 \mathrm{~s}$, where the noise is about 0.5 to $1 \mathrm{BU}$. Because of the influence of the SAA on the dark measurements, other space instruments flying in almost the same low Earth orbit, such as the Ozone Monitoring Instrument (OMI) or SCIAMACHY (Scanning Imaging Absorption Spectrometer for Atmospheric Chartography), do not use those calibration data $[\underline{32}, \underline{33}]$.

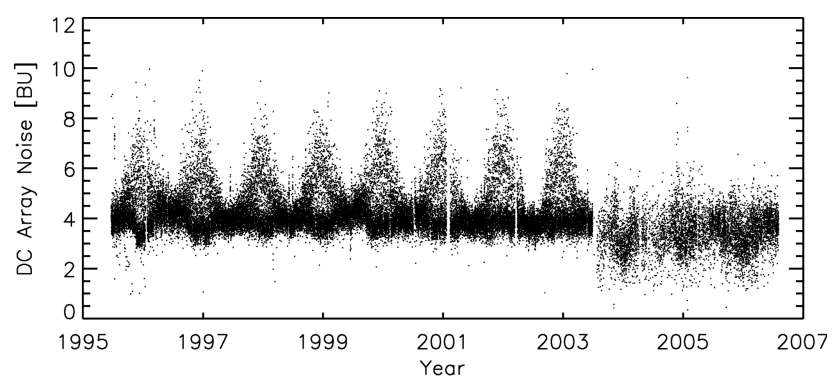

(a)

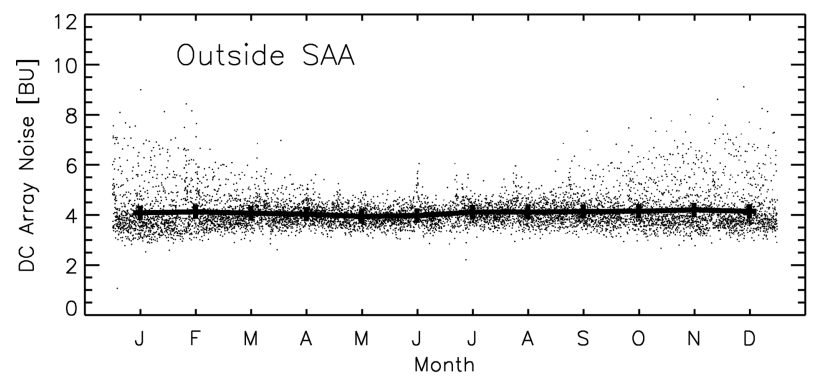

(b)

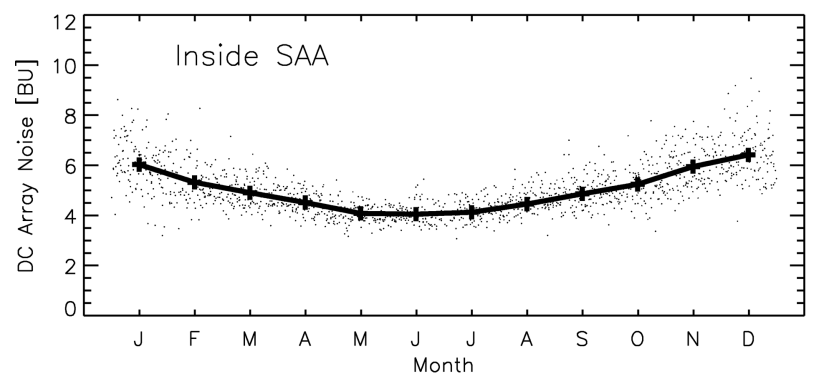

(c)

Fig. 11. (a) Dark current noise for time pattern 320 as a function of time from 1995 to 2006. (b) Noise values in 1997, measured outside the SAA region, plotted as a function of month in 1997. (c) Noise values in 1997, measured inside the SAA region, plotted as a function of month in 1997. Thick curves in (b) and (c) denote monthly mean values.

\section{Pixel-to-Pixel Gain Correction}

One of the characteristics of a diode detector array is the slightly different sensitivity of each individual detector pixel. To correct for this effect, the determination of so-called pixel-to-pixel gain (PPG) correction factors is required. They are assessed by illuminating the detector arrays by internal LEDs, which are basically monochromatic and illuminate the detectors directly, i.e., not through any dispersing element. The monitored detector signal is then a superposition of a smoothly varying signal caused by the LED characteristics and a rapidly changing structure, the pixelto-pixel variation [3]. The correction spectra for each of the four channels are defined as

$$
c_{i}=\frac{S_{i}^{\text {smooth }}}{\overline{S_{i}^{\mathrm{LED}}}},
$$

where $c_{i}$ is the correction factor of detector pixel $i$, $S_{i}^{\mathrm{LED}}$ is the mean value of several consecutive LED 
measurements, and $S_{i}^{\text {smooth }}$ is the smoothed curve through this averaged measurements. The latter is calculated by means of

$$
S_{i}^{\text {smooth }}=\frac{\sum_{k=-n}^{n} \frac{n-|k|}{n} \times \overline{S_{i+k}^{\mathrm{LED}}}}{\sum_{k=-n}^{n} \frac{n-|k|}{n}},
$$

using a triangle filtering window of width $n=5$. The application of the PPG correction is then simply

$$
S_{i}^{\mathrm{corr}}=S_{i} c_{i},
$$

where $S_{i}$ is the measured signal value of detector pixel $i$ and $S_{i}^{\text {corr }}$ is the corrected value.

The LED spectra, which were obtained typically in monthly intervals from 1995 to 2003, as well as the PPG correction spectra, are stored in the GOME calibration parameter database. Because of the failure of the ERS-2 onboard data-storage system in 2003, the LED measurements from 2004 to 2006 are limited to two or three spectra per year. The LED spectra show a considerable linear decrease in time of $20 \%$ to $30 \%$ from 1995 to 2006 . The percentage decrease as a function of wavelength is depicted in Fig. 12, where the ratio of one LED measurement per year, except 2004, to one selected spectrum from August 1995 is shown. The decrease, which is due to the degradation of the LED's brightness itself, is more or less homogeneous over the whole wavelength range of each channel. It is maximum in channel $1(>30 \%)$ and minimum in channel $3(\sim 20 \%)$. Additional differences between individual LED measurements may arise due to inhomogeneous ice-layer buildups on the detectors.

As the calculated pixel-to-pixel gain correction factors are around $0.02 \%$, the absolute radiance correction is not very large. Nevertheless, it may not be negligible in those wavelength regions of the weak absorbers, e.g., bromine oxide (BrO). Although PPG cancels out to a large extent in the ratio of earthshine radiance to solar irradiance, which is

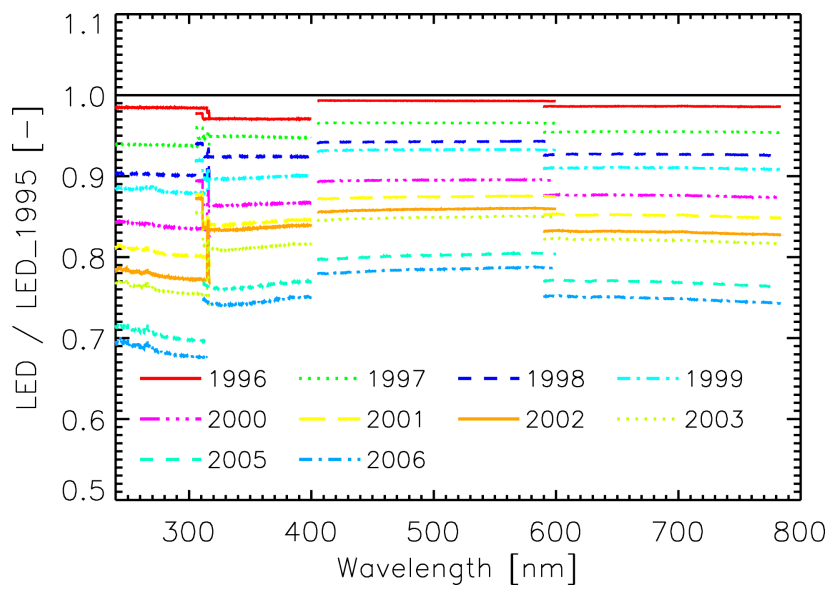

Fig. 12. (Color online) Ratios of the LED Spectra from January 1996 to 2006 to the corresponding spectrum from August 1995. The year 2004 is missing. used in the trace gas retrieval, an up to $10 \%$ effect may remain due to spectral shift. The solar spectra are Doppler-shifted by up to $\sim 0.08$ pixel; in addition, there is some orbital dependence of the wavelength calibration. For this reason, the long-term analysis of the PPG correction was limited to four narrow spectral bands covering the following important wavelength regions: $290-300 \mathrm{~nm}$ (channel $1, \mathrm{O}_{3}$ profile retrieval), $345-356 \mathrm{~nm}$ (channel $2, \mathrm{BrO}$ column retrieval), $420-440 \mathrm{~nm}$ (channel $3, \mathrm{NO}_{2}$ column retrieval), and 750-770 nm (channel 4, cloud detection in $\mathrm{O}_{2}$ A-band).

Figure 13 shows the RMS of the PPG correction over the four spectral bands defined above. It is around $6.5 \times 10^{-4}$ in channel 1 and around $2-3 \times$ $10^{-4}$ in channels 2,3 , and 4 . The RMS in channel 1 remains roughly stable over the entire period, whereas the RMS in channel 2 in the region of the
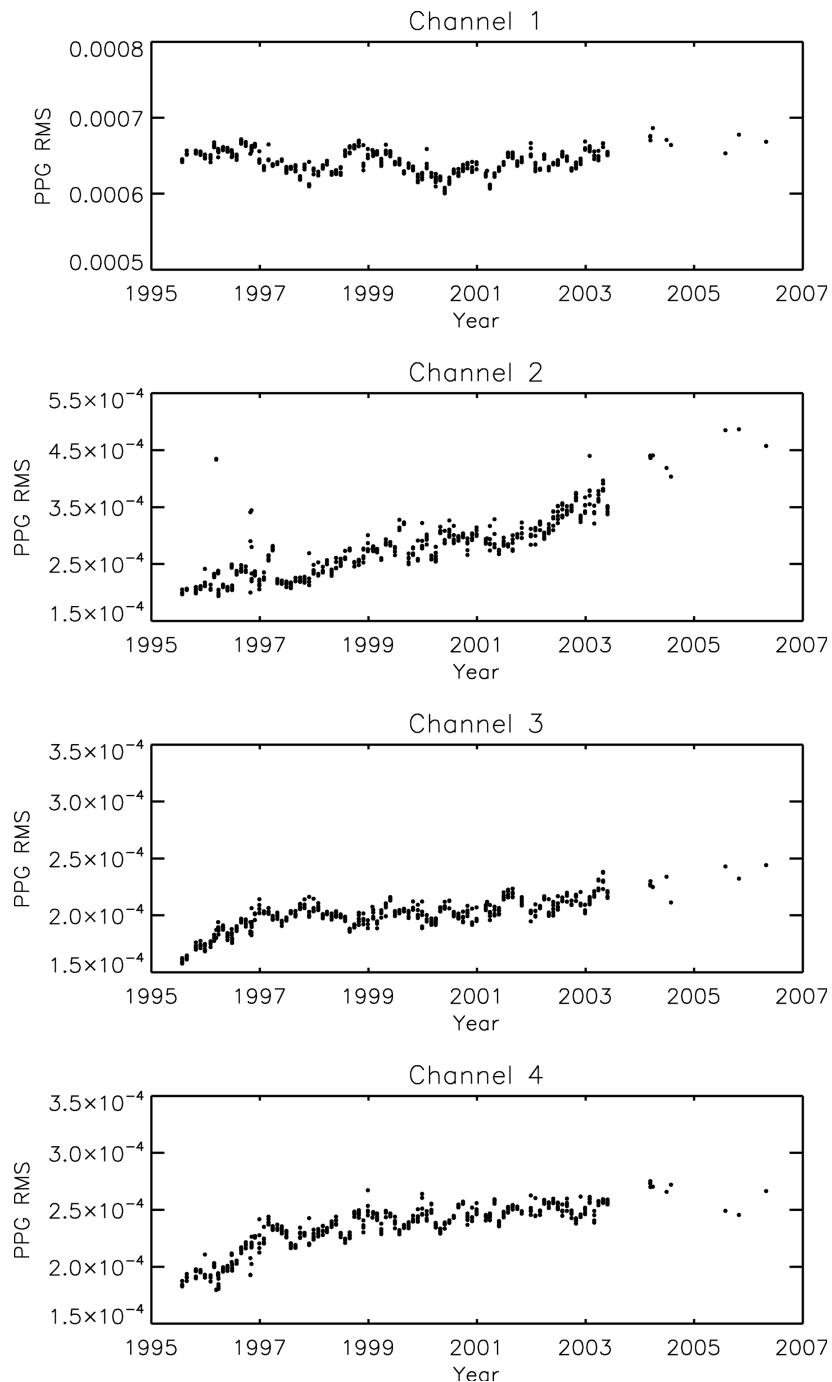

Fig. 13. Time series of the RMS of the PPG correction for four narrow spectral bands from top to bottom: 290 to $300 \mathrm{~nm}$ in channel 1 (used for ozone profile retrieval), 345 to $356 \mathrm{~nm}$ in channel 2 (BrO column amount retrieval), 420 to $440 \mathrm{~nm}$ in channel $3\left(\mathrm{NO}_{2}\right.$ retrieval), and 750 to $770 \mathrm{~nm}$ in channel 4 (cloud detection using oxygen A-band). 
$\mathrm{BrO}$ retrieval increased considerably by a factor of 2 from 1995 to 2006. The increase of the RMS in channels 3 and 4 is about 50\%. That implies that the amplitudes of the correction spectra strongly increased during the lifetime of GOME, which is due to a significantly increasing difference in sensitivity between the individual detector pixels. The change of the distribution of the PPG correction factors in channel 2 (whole channel now) between 1995 and 2003 is depicted in Fig. 14. The results from above are confirmed as the distribution becomes wider and the range increases. Note that the LEDs emit in the red; it is not known how far this is representative for the UV response. If the PPG would be mainly due to a quantum-efficiency effect, the LED measurements may not provide an accurate measure of the true PPG in channels 1 and 2. Nevertheless, Fig. 14 indicates that the increase in PPG is not due to just a few strongly degraded pixels, but affects the detector arrays as a whole.

\section{Summary and Conclusions}

A detailed analysis of 11 years of GOME/ERS-2 inflight calibration parameters has been presented. It is used to monitor the long-term stability and performance of the instrument and its measurements, in order to ensure the high-quality satellite data that are urgently needed for an accurate global climate monitoring. In the framework of the GDP level 0to-1 processor update in 2006 , a complete reprocessing of the entire GOME data set has taken place. A fully homogeneous calibration data set is now available, covering 11 years from 1995 to 2006, which contains numerous diagnostic parameters.

By means of the daily solar irradiance measurements, the degradation of the GOME sensor was monitored. In channels 1 and 2 , the intensity decreased by $70 \%$ to $90 \%$ and $35 \%$ to $65 \%$, respectively. The degradation in channels 3 and 4 is lower, and became significant in 2001. A transmission increase in the early part of the GOME mission may be related to changes in the multilayer coating of the dichroic filter, which

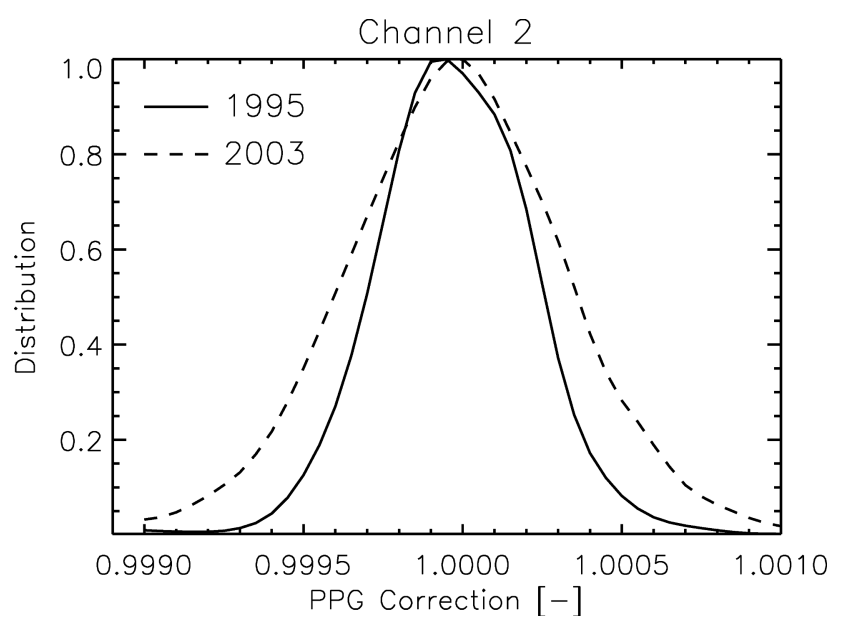

Fig. 14. PPG correction factors distribution in channel 21995 (solid curve) and 2003 (dashed curve). separates both channels. The main degradation has been explained in terms of deposits on the scan mirror. The signals of the three PMDs show qualitatively a similar degradation as the corresponding channel wavelengths. A quantitative analysis of polarization calibration constants called $Q$-factors yields a slightly differential degradation between PMDs and the corresponding channel signals.

The stability of the spectral calibration was inferred by monitoring both the difference between the exact and calculated wavelength of the emission lines and the variability of all calculated GOME wavelengths for a fixed predisperser temperature. The latter is very stable and lies between 0.0015 and $0.004 \mathrm{~nm}$ over the whole spectral range, except for the beginning of channel 3, due to a lack of suitable emission lines. Analyzing the correlation between wavelength and predisperser temperature yields a very complex interaction, which depends on the spectral position on the detector.

Detector properties, such as dark signal and pixelto-pixel gain, show a steady increase over the 10 years analyzed. This occurs on the detectors as a whole, rather than just in a few bad pixels. A linear increase of the dark signal of $3.5-4.5 \mathrm{BU} / \mathrm{s}$ in 10 years was found. The PMD zero offsets show a small increase of $0.5 \%$ to $1 \%$ up to 2005 . The noise of the detector pixel readouts and the PMD noise level showed a distinct seasonal cycle until June 2003. We identify this as the influence of the SAA region (from which few measurements were available after 2003). For this reason, the recently updated version 4.0 of the GDP level 0-to-1 processor includes an algorithm to automatically discard anomalous calibration measurements, such as the ones over the SAA region; calibration packets are checked for the PMD noise, whether this value is below or above a certain threshold. The pixel-to-pixel gain, derived from LED measurements, increased in channel 2 by $100 \%$ and in channels 3 and 4 by about $50 \%$.

This extensive study of the long-term behavior of numerous GOME in-flight calibration parameters underlines the importance of this work for improving instrument calibration algorithms. We would recommend applying this kind of analysis to recent backscatter instruments, such as SCIAMACHY on ENVISAT and GOME-2 on METOP, to ensure high-quality satellite data as a contribution to global climate monitoring.

We would like to thank the reviewers; this paper has benefited from their careful comments. This work was partially funded by ESA/ESRIN (MMFI/ D-PAF contract).

\section{References}

1. J. P. Burrows, M. Weber, M. Buchwitz, V. V. Rozanov, A. Ladstädter-Weissenmayer, A. Richter, R. de Beek, R. Hoogen, K. Bramstedt, K.-U. Eichmann, M. Eisinger, and D. Perner, "The Global Ozone Monitoring Experiment (GOME): mission concept and first scientific results," J. Atmos. Sci. 56, 151175 (1999). 
2. D. Loyola, W. Balzer, B. Aberle, M. Bittner, K. Kretschel, H. Muehle, T. Ruppert, C. Schmid, S. Slijkhuis, R. Spurr, W. Thomas, T. Wieland, and M. Wolfmueller, "Ground segment for ERS-2/GOME sensor at the German D-PAF," in 3rd ERS Scientific Symposium, Special Publication SP-414 (European Space Agency, 1997), Vol. II, pp. 591-596.

3. B. Aberle, W. Balzer, A. von Bargen, E. Hegels, D. Loyola, and R. Spurr, "GOME level 0-to-1 algorithms description," Tech. Rep. ER-TN-DLR-GO-022 (German Aerospace Center, 2002), Issue/Revision 5/B, http://earth.esrin.esa.it/pub/ESA DOC/GOME/.

4. M. Coldewey-Egbers, S. Slijkhuis, B. Aberle, and D. Loyola, "ERS-2 GOME GDP level 0-to-1 improvements, software verification report," Tech. Rep. ER-TN-DLR-GO-050 (German Aerospace Center, 2006), Issue 1.

5. G. H. Mount, R. W. Sanders, and J. W. Brault, "Interference effects in reticon photodiode array detectors," Appl. Opt. 31, 851-858 (1992).

6. M. Weber, J. P. Burrows, and R. P. Cebula, "GOME solar UV/ VIS irradiance measurements between 1995 and 1997: first results on proxy solar activity studies," Sol. Phys. 177, 6377 (1998).

7. M. van Roozendael, D. Loyola, R. Spurr, D. Balis, J.-C. Lambert, Y. Livschitz, P. Valks, T. Ruppert, P. Kenter, C. Fayt, and C. Zehner, "Ten years of GOME/ERS-2 total ozone data. The new GOME data processor (GDP) version 4:1. Algorithm description," J. Geophys. Res. 111, D14311 (2006).

8. GOME yearly anomaly reports, http://earth.esa.int/ers/gome/ performance/.

9. R. P. Cebula, H. Park, and D. F. Heath, "Characterization of the Nimbus-7 SBUV radiometer for the long-term monitoring of stratospheric ozone," J. Atmos. Ocean. Technol. 5, 215227 (1988).

10. A. Dehn, "Long term monitoring of GOME diffuser reflectivity and dark signal analysis," Tech. Rep. ERS2-GO-DDS-TN-006 (European Space Agency-European Space Research Institute, 2001), Issue 1.0.

11. R. Snel, "In-orbit optical path degradation: GOME experience and SCIAMACHY prediction," in Proceedings of the ERS-ENVISAT Symposium, Special Publication SP-461 (European Space Agency, 2001), CD-ROM.

12. J. M. Krijger, C. P. Tanzi, I. Aben, and F. Paul, "Validation of GOME polarization measurements by method of limiting atmospheres," J. Geophys. Res. 110, D07305 (2005).

13. I. Aben, M. Eisinger, E. Hegels, and C. Tanzi, "GDAQI final report," Tech. Rep. TN-GDAQI-003 SR/2000 (European Space Agency/ESRIN, 2000), http://wdc.dlr.de/sensors/gome/ degradation_files/literature/gdaqi_final.pdf.gz.

14. J. M. Krijger, I. Aben, and J. Landgraf, "CHEOPS-GOME: WP2.1: Study of instrument degradation," Tech. Rep. SRON-EOS/RP/05-018 (European Space Agency, 2005).

15. R. Spurr, D. Loyola, W. Thomas, W. Balzer, E. Mikusch, B. Aberle, S. Slijkhuis, T. Ruppert, M. van Roozendael, J.-C. Lambert, and T. Soebijanta, "GOME level 1-to-2 data processor version 3.0: a major upgrade of the GOME/ERS-2 total ozone retrieval algorithm," Appl. Opt. 44, 7196-7202 (2005).

16. D. Balis, J.-C. Lambert, M. van Roozendael, R. Spurr, D. Loyola, Y. Livschitz, P. Valks, V. Amiridis, P. Gerard, J. Granville, and C. Zehner, "Ten years of GOME/ERS2 total ozone data. The new GOME data processor (GDP) version 4: 2. Groundbased validation and comparisons with TOMS V7/V8," J. Geophys. Res. 112, D07307 (2007).

17. J. E. Murray, Atlas of the spectrum of a platinum/chromium/ neon hollow-cathode reference lamp in the region
240-790 nm, Final Report to European Space Agency (John Wheaton Associates, 1994).

18. European Space Agency, The Global Ozone Monitoring Experiment (GOME) Users Manual, F. Bednarz, ed., Tech. Rep. SP1182 (European Space Agency, 1995).

19. W. H. Press, S. A. Teukolsky, and W. T. Vetterling, Numerical Recipes in Fortran 77 (Cambridge U. Press, 1992).

20. M. Coldewey-Egbers, S. Slijkhuis, and D. Loyola, "ERS-2 GOME long term monitoring of GOME calibration parameters," Tech. Rep. ER-TN-DLR-GO-0000 (DLR, 2006), Issue 2/A.

21. D. Loyola, W. Thomas, Y. Livschitz, T. Ruppert, P. Albert, and R. Hollmann, "Cloud properties derived from GOME/ERS-2 backscatter data for trace gas retrieval," IEEE Trans. Geosci. Remote Sens. 45, 2747-2758 (2007).

22. J. H. G. M. van Geffen, "Wavelength calibration of spectra measured by the Global Ozone Monitoring Experiment: variations along orbits and in time," Appl. Opt. 43, 695705 (2004).

23. R. O. Green, "Spectral calibration requirement for Earth-looking imaging spectrometers in the solar-reflected spectrum," Appl. Opt. 37, 683-690 (1998).

24. A. von Bargen and S. Slijkhuis, "Wavelength calibration of GOME spectra utilising cross-correlation," in ESAMS '99European Symposium on Atmospheric Measurements from Space, Publication WPP-161 (European Space Agency, 1999), pp. 709-713.

25. J. H. G. M. van Geffen and R. F. van Oss, "Wavelength calibration of spectra measured by the Global Ozone Monitoring Experiment by use of a high-resolution reference spectrum," Appl. Opt. 42, 2739-2753 (2003).

26. G. Jaross, A. Krueger, R. P. Cebula, C. Seftor, U. Hartmann, R. Haring, and D. Burchfield, "Calibration and postlaunch performance of the Meteor 3/TOMS instrument," J. Geophys. Res. 100, 2985-2995 (1995).

27. E. Hilsenrath, D. E. Williams, R. T. Caffrey, R. P. Cebula, and S. J. Hynes, "Calibration and radiometric stability of the Shuttle Solar Backscatter Ultraviolet (SSBUV) Experiment," Metrologia 30, 243-248 (1993).

28. R. P. Cebula, E. Hilsenrath, P. W. DeCamp, K. Laamann, S. Janz, and K. McCullough, "The SSBUV experiment wavelength scale and stability: 1988 to 1994," Metrologia 32, 633-636 (1995).

29. A. Dehn, "GOME diffuser and dark signal trends (June 1995June 2002)," (European Space Agency, 2002), http://earth.esa .int/ers/instruments/gome/gome_quality/ diffuserdark_upjune02f55.html.

30. J. R. Heirtzler, "The future of the South Atlantic anomaly and implications for radiation damage in space," J. Atmos. Sol. Terr. Phys. 64, 1701-1708 (2002).

31. X. Liu, K. Chance, C. E. Sioris, R. J. D. Spurr, T. P. Kurosu, R. V. Martin, and M. J. Newchurch, "Ozone profile and tropospheric ozone retrievals from the Global Ozone Monitoring Experiment: algorithm description and validation," J. Geophys. Res. 110, D20307 (2005).

32. M. Dobber, "OMI in-flight calibration plan," Tech. Rep. PLOMIE-KNMI-393 (Royal Netherlands Meteorological Institute, 2003), Issue 2.

33. S. Slijkhuis, "ENVISAT-1 SCIAMACHY level 0-to-1c processing algorithm theoretical basis document," Tech. Rep. ENV-ATB-DLR-SCIA-0041 (German Aerospace Center, 1999), Issue 1, http://earth.esa.int/services/sample_products/ sciamachy/documentation/SCIAMACHY/. 